\title{
1 A Modified IEEE 802.11 MAC for Optimizing Broadcasting 2 in Wireless Audio Networks
}

\author{
3 Christos Chousidis ${ }^{1}$ (D) Ioana Pisca $^{2} \cdot$ Zhengwen Huang $^{2}$ \\ Received: 16 December 2018 / Revised: 9 March 2019 / Accepted: 31 May 2019 \\ (c) Springer Science+Business Media, LLC, part of Springer Nature 2019
}

\section{Abstract}

The use of network infrastructures to replace conventional professional audio systems is a rapidly increasing field which is expected to play an important role within the professional audio industry. Currently, the market is dominated by numerous $\mathbf{A Q 1}$ proprietary protocols which do not allow interoperability and do not promote the evolution of this sector. Recent standardization actions are intending to resolve this issue excluding, however, the use of wireless networks. Existing wireless networking technologies are considered unsuitable for supporting real-time audio networks, not because of lack of bandwidth but due to their inefficient congestion control mechanisms in broadcasting. In this paper, we propose an amendment of the IEEE 802.11 MAC that improves the performance of the standard for real-time audio data delivery. The proposed amendment is offering a solution for the balancing of data flow density in wireless ad-hoc networks for a multi-broadcasting environment. It is based on two innovative ideas. First, it provides a protection mechanism for broadcasting and second, it replaces the classic congestion control mechanism, based in random backoff, with an alternative traffic adaptive algorithm, designed to minimize collisions. The proposed MAC is able to operate as an alternative mode allowing regular Wi-Fi networks to coexist and interoperate efficiently with audio networks, with the last ones being able to be deployed over existing wireless network infrastructures.

Keywords Audio networks · Congestion control algorithm · CTS-to-Self · Exclusive backoff number allocation algorithm $\cdot \mathrm{H}$-EBNA $\cdot$ MAC modification $\cdot$ Wireless audio networks · Broadcasting

Christos Chousidis

christos.chousidis@uwl.ac.uk

1 School of Computing and Engineering, University of Went London, St Mary's Road, W5 5RF London, UK

2 Department of Electronics and Computer Engineering, Brunel University, Kingston Lane, UB8 3PH London, UK 


\section{Introduction}

Today the rapid growth of networking technology, along with the dominance of digital audio, makes essential the implementation of audio networks in the majority of the professional audio applications. The term "audio networking" is introduced to describe the usage of a network infrastructure for the delivery of digital audio data, between audio sources and audio processing devices, within sound installations such as recording and broadcasting studios and live music stage systems. Often, audio networks are wrongly considered as networks for generic audio delivery. In reality, the term "Audio Networks" is used to describe dedicated networking infrastructures that are offering an alternative interconnection for audio systems in live-music scenarios. The concept of such an implementation is described in Fig. 1. This figure shows a live-music system that consists of several sound source, a mixing console, several audio processing units such as equalizers, audio compressors and reverberation processors, a set of stage monitor speakers and a main public address (PA) system.

These devices are interconnected over a local area network and can be considered as nodes that exchanges audio data.

In the past two decades the idea of using data networks for the delivery of audio information has been introduced and a plethora of standards and systems has been proposed with the majority of them based on the Ethernet protocol. [1]. As it is usual with new technologies that have a commercial interest, the

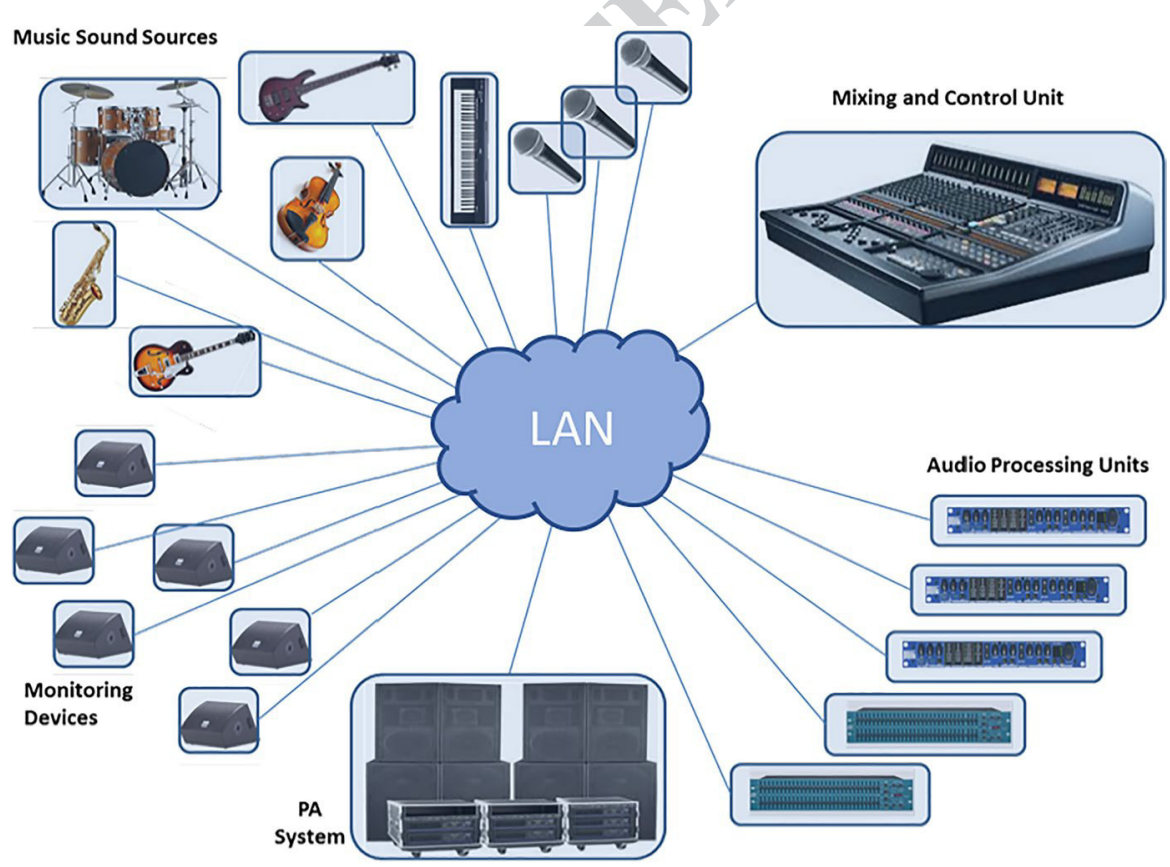

Fig. 1 The live-stage audio networking concept 
lack of common practices and standards has led to the development of numerous proprietary systems lacking the ability to interact between each other. During the last years a standardization effort has been made in order to alleviate this problem [2]. An overview of the professional audio industry today, shows that audio networks are not widespread. This is significantly disproportionate to the general expansion of networking system in other technological fields and shows that significant progress can be made in this sector.

One of the key steps that is expected to help the evolution and spread of audio networks is their migration to the wireless domain. However, this requires wireless networks that will be able to distribute data with a very low packet loss rate and delay. Moreover, they should be able to operate in a multi-broadcasting environment as each member in the network requires data from all other members.

The reliable multiple broadcasting of time sensitive data over wireless networks has not been thoroughly investigated. Research in this field focuses mainly on the cross-layer optimization of single broadcasting streams [3, 4]. Authors in [5] propose a resource allocation solution that improves Quality of Service (QoS) for multimedia wireless transmission and it can be a practical solution for real-time wireless networks. However, this requires a wireless network with relatively high bandwidth. In [6], authors address the problem of delivering a single low-latency multimedia stream in a mobile ad-hoc environment by introducing a cross-layer congestion control strategy. The majority of the above described solutions, however, are video oriented. Authors in [7] present an optimization framework for transmitting high quality audio sequences over wireless links. Their effort however focuses on data encoding and it is a solution for low error audio delivery over hostile wireless environment.

In this paper we develop a custom solution specifically designed to cover the needs in throughput and latency in an audio multi-broadcasting environment. The proposed solution is based on two novel ideas. The first is the development of a protection mechanism in broadcasting, similar to the one that is used in IEEE 802.11 for unicast transmission. The second is the development of a traffic adaptive congestion control algorithm that takes into account the special characteristics of an audio network and is able to eliminate collisions for a finite number of stations (STAs) in an ad-hoc network.

The rest of this paper is organized as follows. In section two, the problems that prevent the implementation of audio networks using existing wireless networking technologies are analyzed. In section three, a novel collision protection mechanism for broadcasting in wireless networks is proposed. This method is tested for multi-broadcasting audio traffic and the results are presented and discussed. In section four, the exclusive backoff number allocation (EBNA) concept is explained and simulated, and its advantages and disadvantages are thoroughly analyzed. In section five, a hybrid-EBNA method for optimal performance is proposed and the comparative results are analyzed and discussed. Finally, in section six the overall conclusions of this work are presented. 


\section{Defining the problem in Wireless Audio Networks}

A white paper released by the technical committee on network audio systems (TC-NAC) of the Audio Engineering Society (AES) in 2009 [8] states that the audio networking sector will benefit from the adoption of wireless networking technologies, but it considers existing wireless protocols incapable of supporting this implementation.

However, it is evident that the successful development of wireless audio networks must be based on existing commercial standards that will be able to provide an efficient and inexpensive solution when it comes to hardware. The most appropriate candidate that fulfils the above requirement is the IEEE 802.11 standard. IEEE 802.11 is a reliable standard that offers the necessary bandwidth for the delivery of a sufficient number of uncompressed audio channels for professional applications.

Audio networks are implemented in an area of few square meters and therefore there is always a good radio signal level and all nodes are in line-of-sight. Taking this into account, the most efficient way to implement audio networks with multiple audio channels over IEEE 802.11 WLANs is to use an ad-hoc configuration and broadcasting as a delivery method. This is because all other methods require retransmission which is inappropriate for time sensitive data. However, the Medium Access Control (MAC) algorithm [9] that handles broadcasting over 802.11 networks is not designed for the distribution of high-rate, time-sensitive media data. Broadcasting in IEEE 802.11 ad-hoc networks was initially designed for network control purposes. The reliable broadcasting of media data in IEEE 802.11 networks is an interesting research area, nevertheless, the majority of the research related to this subject focuses in reliability issues rather that providing quality of service $[10,11,12]$.

\subsection{Drawbacks of Broadcasting in IEEE 802.11 networks}

Broadcasting is the appropriate method of distributing media data as it can deliver data to multiple users with the minimum occupation of the wireless medium. However, due to the lack of a specific recipient, acknowledgment (ACK) techniques cannot be implemented and thus, broadcasting is unable to provide any kind of packet delivery guarantee. [13].

The medium access control in IEEE 802.11 networks is achieved by using the Carrier Sense Multiple Access, with Collision Avoidance mechanism (CSMA/ CA). However, collisions cannot be totally avoided. In addition, due to the lack of a delivery report in the case of broadcasting, collided packets cannot be identified and recovered $[14,15]$.

The IEEE 802.11 MAC implements a probabilistic method of reducing collisions by assigning random waiting time intervals to all STAs that have a packet for transmission. The values of these waiting time intervals are drawn from a finite set of integers called Contention Window (CW). When a STA detects the 


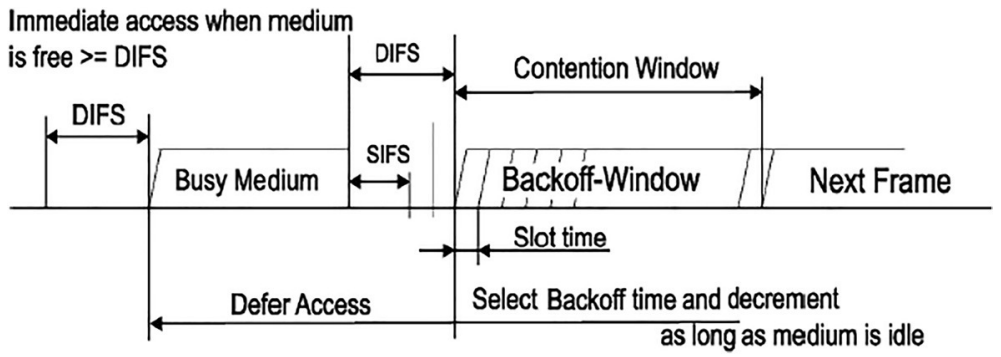

Fig. 2 IEEE 802.11 basic access method, (IEEE 802.11 Working Group, 2007)

wireless medium to be idle for a time frame equal to a DCF Inter-Frame Space (DIFS) and it has a packet for transmission, the MAC algorithm assigns to it an additional backoff time (Fig. 2) that is defined by Eq. 1.

$$
\text { Backof }_{\text {Time }}=\operatorname{INT}[\text { CWxRandom }(0,1)] \times \text { aSlotTim }
$$

Random $(0,1)$ is a pseudo-random number between 0 and 1 , drawn using a uniform distribution, and aSlotTime is a value defined by the protocol.

In a unicast transmission, when an ACK message is not received after the transmission of a data packet, the CW increases exponentially, and a retransmission attempt is performed. This process is repeated until the packet is successfully delivered or the $\mathrm{CW}$ reaches its maximum size. The $\mathrm{CW}$ is described by the CWmin and $C W \max$ values and its size is defined from the relation $C$ Wvalues $=2^{\mathrm{x}}-1$. The initial value of ' $x$ ' is an integer which is defined by the protocol and it can be increased up to 10 . That means that the maximum size of $\mathrm{CW}$ can be $C W_{10}=1023$.

In the case of broadcasting, the size of CW remains constantly minimum as long as the ACK technique cannot be implemented.

In addition to the above standard technique for the network's arbitration, the optional technique of network allocation vector (NAV) can be also used. NAV is a technique that distributes information related to the time that the medium will be occupied by the STA that recently got access to it. This again, is implemented only in unicast transmission and it is achieved by the exchange of Request-to-Send, Clear-to-Send control messages (RTS/CTS). This is actually a dialogue between the sender and the receiver that secures a reception readiness from the recipient's side. Although this is a message exchange between two specific STAs, RTS and CTS are broadcasting messages and the information they contain regarding the network's allocation is distributed across the entire network. STAs that receive these messages are deferring transmission for the designated time period, reducing this way the risk of dropped and collided packets. When the transmission of a RTS is not possible, an alternative CTS message sent from a STA with destination its own address, can be used to distribute NAV information. This type of "blind" CTS message is called CTS-to-Self. This method is used for collision protection from the IEEE 802.11 standard only for mixed-mode environments where extended rate physical (ERP$802.11 \mathrm{~g}$ ) and/or high throughput (HT-802.11n) STAs coexist with legacy 802.11 technologies [16]. 
The fact that in broadcasting ACK cannot be implemented results in the size of $\mathrm{CW}$ being always its minimum value. When the number of broadcasting STAs in the network increases, the probability of two or more STAs to select simultaneously the same backoff number is also increased [17]. In an audio multiple-broadcasting environment the likelihood of experiencing this problem is even higher. This is because the heavy payload created by audio data it forces STAs to intensively attempt access to the wireless medium. In such a case a significant number of packets is lost as collisions in broadcasting cannot be identified and collide packets cannot be recovered.

\subsection{Collision probability in a multi-broadcasting environment}

In broadcasting over 802.11 networks the $\mathrm{CW}$ remains always in its minimum size (CWmin). All STAs participating in a wireless audio network are constantly producing audio data as they meant to serve musicians within a group that play simultaneously. Therefore, all STAs within an audio network attempt constantly to access the wireless medium [19]. When a STA finds the medium idle for a DIFS, its MAC algorithm, following a uniform distribution, selects a random integer backoff time within a sample space of $[0-C W m i n]$ and assigns this number to the backoff counter. The backoff counter is decremented for every time slot that the medium is sensed idle. If during this countdown the medium becomes busy, the countdown stops and resumes whenever the medium becomes idle again. However, if two or more STAs perform the backoff process concurrently and they select equal backoff numbers, they will all reach zero and broadcast simultaneously causing, therefore, a collision [19]. For the above scenario, the probability $p_{1}$ of a STA to transmit in an arbitrary slot will be:

$$
p_{1}=\frac{1}{C W \min }
$$

Therefore, the probability of a STA not to transmit in an arbitrary slot $p_{2}$ is:

$$
p_{2}=1-\frac{1}{C W m i n}
$$

If a network consists of $n$ number of STAs and at an arbitrary slot the STA $i$ is transmitting, then the number of non-transmitting STAs in the network will be $j=n-1$. In broadcasting over an ad-hoc network all attempt of accessing the medium are independent events [20]. Taking also into account Eq. (3) and the product law of independent events, the probability $p_{2(j)}$ of no other STAs transmitting in this specific slot will be:

$$
\begin{aligned}
& p_{2(j)}=p_{2(1)} \times p_{2(2)} \times \cdots \times p_{2(i-1)} \times p_{2(i+1)} \times \cdots \times p_{2(n)} \\
& p_{2(j)}=\left(1-\frac{1}{C W \text { min }}\right)^{n-1}
\end{aligned}
$$
mit a packet during this slot and it can be expressed as follows: 


$$
p_{(\text {collision })}=1-p_{2(j)}=1-\left(1-\frac{1}{C W \min }\right)^{n-1}
$$

From (5) it is shown that broadcasting in a saturated IEEE 802.11 ad-hoc network is affected only by the number of STAs in the network and the size of CW.

Plotting (5) in Fig. 3, we can see that the probability of collision in a multi-broadcasting environment reaches dramatically high values when the number of broadcasting STAs increases [21]. This is in reality the reason that IEEE 802.11 technologies cannot be used to implement wireless audio networks, despite the available bandwidth.

\subsection{The modified IEEE 802.11 MAC algorithm}

The aim of the amendment of the IEEE 802.11 MAC algorithm, proposed in this paper is to resolve the collision problem and thus to allow the implementation of this technology in wireless audio networks. This amendment consists of two core innovative ideas. The first one is to develop a protection mechanism for broadcasting and the second, to design an alternative congestion control mechanism that eliminates collisions. The above modifications are designed to work together and also to ensure the interoperability between the classic and modified MAC algorithm when they coexist in the same WLAN.

\section{A collision protection mechanism for reliable broadcasting}

The collision protection mechanism proposed in this work is based on the amended use of CTS-to-Self message. This is achieved by two key modifications.

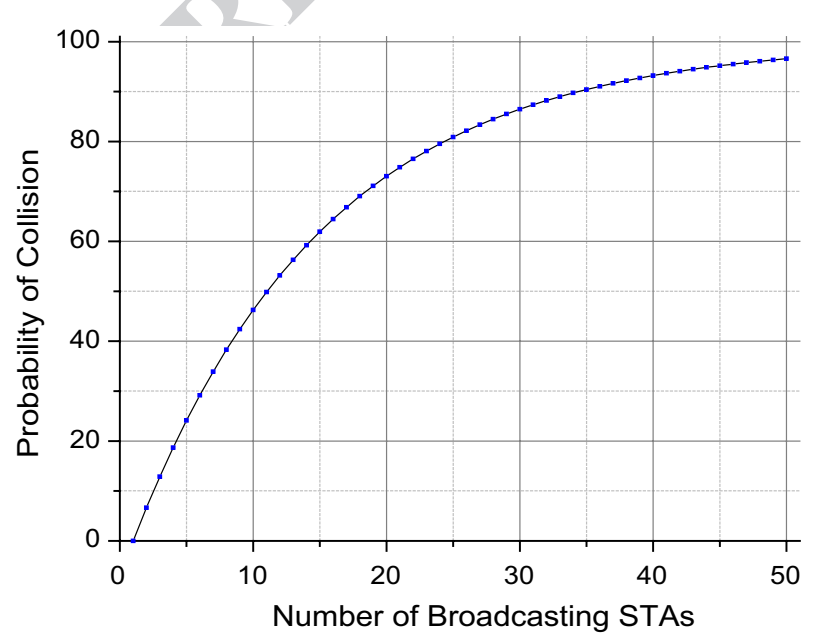

Fig. 3 Probability of collision in broadcasting, $(\mathrm{CW}=15)$ 
In the first modification, the congestion control algorithm was programed to add a CTS-to-Self transmission prior to each data packet transmission [22]. Therefore, in this amended MAC, when a STA successfully completes the random backoff process and gains access to the medium, it sends a CTS message with NAV information to its own address. This informs the rest of the STAs for the time the network will be occupied, in order to avoid unnecessary attempts to access it. In addition, when two or more STAs complete their backoff process simultaneously, according to the case described in section II-B, the inevitable collision will take place between the CTSto-Self messages rather than the data packets. This causes a significantly shorter jam in the network (Fig. 4) and therefore increases throughput. In the case that no collision occurs, the same STA regains immediately access to the network by waiting only for a SIFS and successfully broadcasts the packet, following the procedures described in the original IEEE 802.11 protocol.

In the second modification the content of the CTS-to-Self is also reprogrammed. As it was mentioned earlier in this paper, this control message is originally used for protection when legacy 802.11 technologies are identified in a network and it is broadcasted using the lowest possible rates. In our case, the function that generates this message is reprogrammed in order to always adjust with the current data packet transmission rate, avoiding this way to slow down the entire wireless network.

\subsection{CTS-to-Self modification (simulation and results)}

This study is performed using OPNET Modeler 17.1. The simulation is based on the IEEE $802.11 \mathrm{~g}$ standard. The configuration of the network is ad-hoc and the bit rate is set at $54 \mathrm{Mbps}$. The population of the network is dynamic, starting from 5 STA and gradually increasing to 60 STAs. The data in each STA are created using a packet generator that produces a data rate of $256 \mathrm{Kbps}$. The final broadcasting

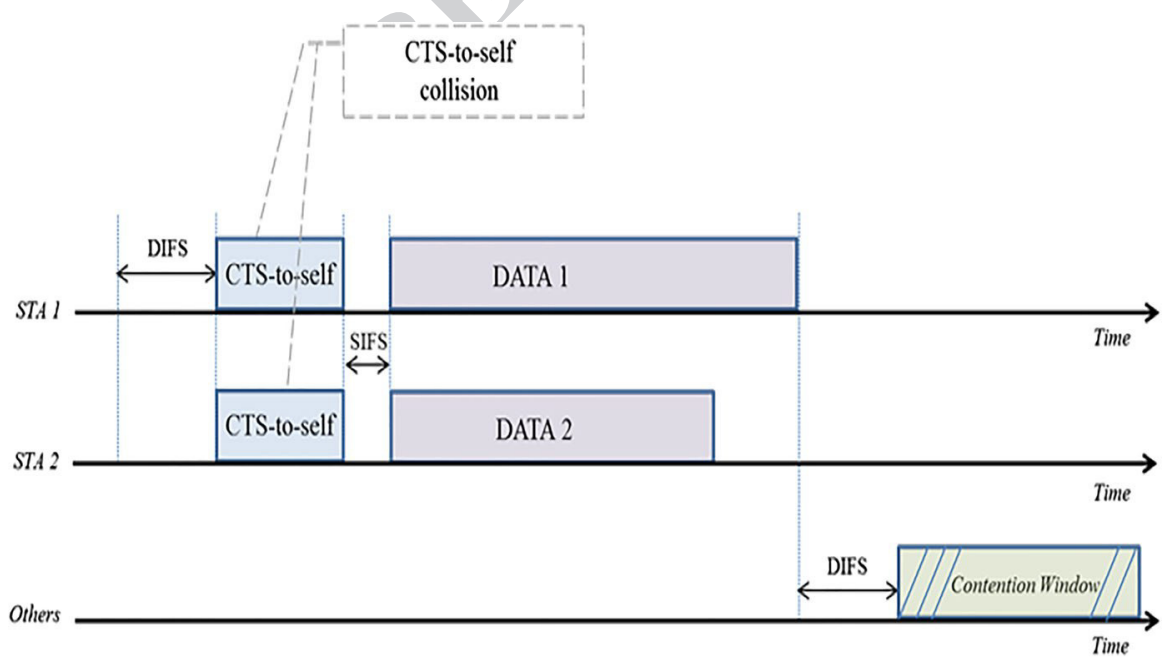

Fig. 4 CTS-to-Self collision 
rate is measured to be $320 \mathrm{Kbps}$. This increase is caused by the MAC overhead. The network is considered saturated and the generation of data load remains constant in all scenarios. However, for each increase of the population three different scenarios are tested using variations of packet size. This is because, for a given data load, the packet size is directly affecting the number of attempts accessing the medium and therefore the number of additional CTS-to-Self messages that are broadcasted. The packet sizes used for this study are 2048, 1024 and 512 bytes. All simulations are performed with both the modified and the classic 802.11 MAC, for comparison reasons. The collected statistics are:

- Throughput

- Number of Collisions per STA

- Overall End-to-End Delay

The results are analytically presented and discussed in [22]. Here we only present results for the 2048 bytes packet size, where we experience the higher throughput improvement. As it is shown in Fig. 5, a constant improvement of throughput is observed in all cases, when the modified 802.11MAC is used.

However, when the number of broadcasting STAs increases beyond 40, the modified MAC is able to handle this traffic, contrary to the classic 802.11 MAC which experiences significant data losses.

In Fig. 6 it is shown that the modified MAC causes an increase of the Endto-End delay which nevertheless remains in acceptable levels. This is expected, and it is caused by the additional traffic that enters in the network from the use of CTS-to-Self messages.

Figure 7 shows the average number of collisions per STA, as the number of STAs increases. We can see here that, although the number of collisions

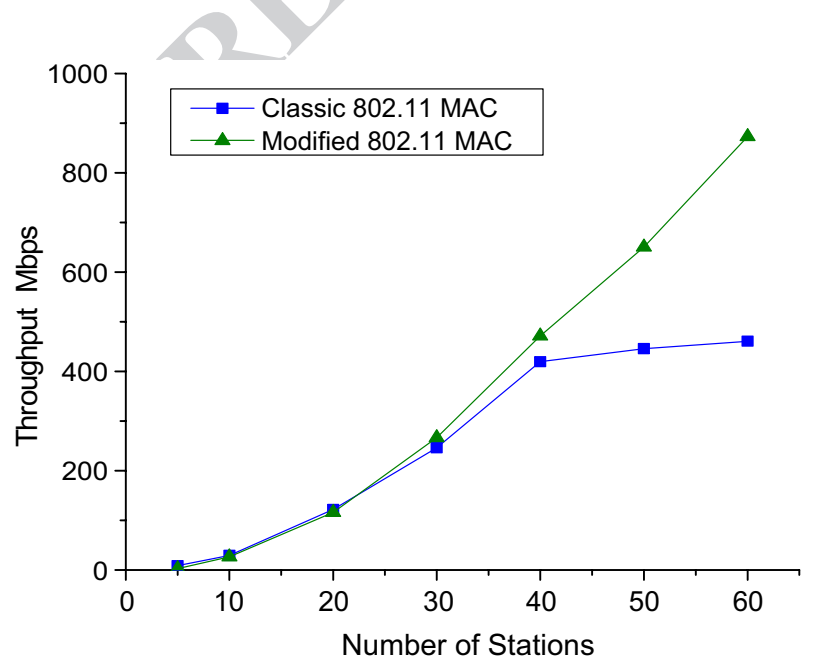

Fig. 5 Throughput performance for 2048 bytes, packet size 


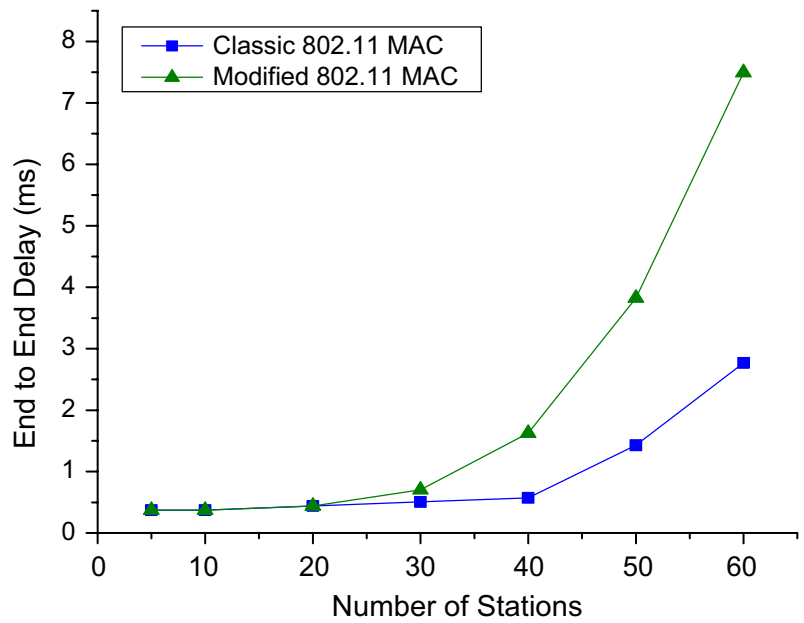

Fig. 6 End-to-end delay for 2048 bytes packet size

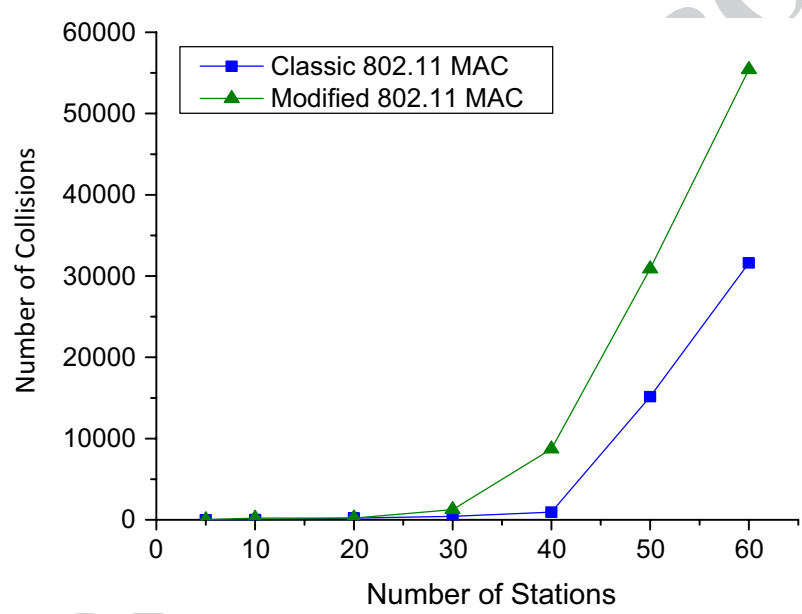

Fig. 7 Average number of collisions per STA for 2048 bytes packet size

271 increases, as the number of broadcasting STAs increases throughput performance 272 also increases.

273 This is because most collision occurs between CTS-to-Self messages rather 274 than data packets and, as it was mentioned earlier, this reduces the negative effect 275 of collisions. 


\section{The EBNA concept}

Using the collision protection mechanism proposed in section three we have a significant decrement of collision rate. However, some collisions will always happen. This is due to the nature of the congestion control mechanism of IEEE 802.11 standard, and most specifically to the random backoff process. In order to deal with this issue, an alternative congestion control mechanism is proposed in this section. The design of this mechanism is based on the fact that audio networks have by nature some specific characteristics. These are mainly the finite and controllable population of the STAs and also of the spatial limitations of the network.

The proposed mechanism replaces the random way that the backoff numbers are assigned in broadcasting packets in the classic 802.11 MAC by assigning exclusive pairs of numbers to different STAs in the network. The operating principle of this Exclusive Backoff Number Allocation (EBNA) algorithm is described as follows:

Assume an ad-hoc network where each STA upon joining the network obtains a STA ID (STID). Let us also assume that each STA knows the overall number of STAs (No_of_STAs) in this network at any given time. In this case we define the size of the $\mathrm{CW}$ as:

$$
C W=2 \times\left(N o \_o f \_S T A s\right)
$$

We also divide this $\mathrm{CW}$ in two groups according to the following rule:

$$
\text { group } 1 \leq \frac{\text { No_of_STAS }}{2}
$$

$$
\text { group } 2>\frac{\text { No_of_STAs }}{2}
$$

When a STA has a packet to transmit and attempts to access the medium, the EBNA algorithm assigns to a variable called GRP, a random value between 1 and 2 using a uniform distribution. The purpose of this process is for the algorithm to randomly select for the next steps one of the two groups defined in (7) and (8). When GRP $=1$, the group 1 is selected and when GRP $=2$, the group 2 is selected. In the first case, the algorithm assigns to the STA a backoff number equal to its STID. In the second case the algorithm assigns to the STA a backoff number which is symmetrically

$$
\begin{aligned}
& \mathrm{CW}=20 \\
& \text { Number of STAs }=10 \\
& \text { STID }=2 \square \\
& \text { STID }=6
\end{aligned}
$$

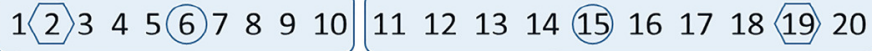

Contention Window

Fig. 8 The EBNA implementation, (an example for STID =2 and STID=6) 
Journal of Network and Systems Management

Table 1 Traffic generation parameters

\begin{tabular}{ll}
\hline Attributes & Values \\
\hline Start time & Normal distribution $(1,0.01)$ \\
On-state & $0.25 \mathrm{~s}$ \\
Off-state & $0.25 \mathrm{~s}$ \\
Interarrival time & Constant distribution $(24.3 \mathrm{~ms})$ \\
Packet size & 2200 bytes
\end{tabular}

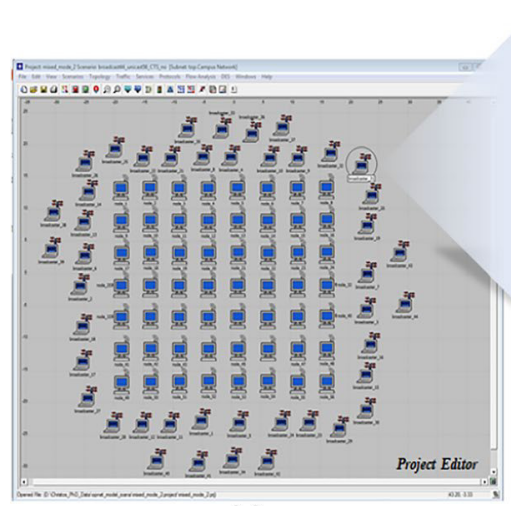

(a)

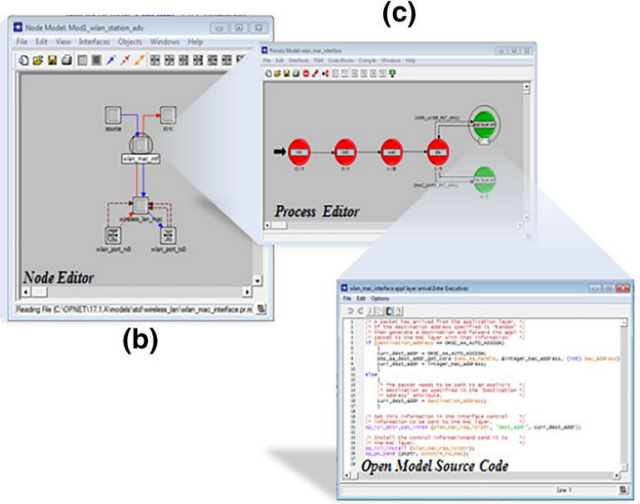

(d)

Fig. 9 OPNET Modeler hierarchical architecture: A 56 audio STAs scenario, (a project editor, b node editor, $\mathbf{c}$ process editor, $\mathbf{d}$ code editor)

opposite to its STID number within the CW, as it is shown in Fig. 8. As we can see, in all cases a STA with a specific STID that tries to access the medium, will obtain a backoff value which will randomly retrogress between a unique pair of numbers, exclusively allocated to this STA. The allocation of backoff slots for each STA is described in the algorithm below:

$$
\text { for } G R P=1 \quad \text { Backof }_{\text {Slots }}=S T I D
$$

$$
\text { for } \left.G R P=2 \quad \text { Backof } f_{\text {slots }}=[(\text { No_of_STA }) \times 2)-S T I D\right]+1
$$

Taking into account that this allocation is based on a uniform distribution and also that the possible values of backoff slots are complimentary within the $\mathrm{CW}$, this process secures fairness in the network. The complimentary values of backoff slots results on an equal average waiting time to all STAs, in the long run. For each STA, the average number of backoff slots will be equal to $\mathrm{CW} / 2$, as it is shown in the example in Fig. 8. 


\subsection{EBNA (simulation and results)}

The simulation characteristics of the proposed EBNA algorithm are similar to those described in section III-A. The ad-hoc network spans in a surface of $30 \times 40 \mathrm{~m}$ with the wireless STAs randomly located within it. The population of the network is again dynamic, starting from 10 STAs and gradually increasing to 70 STAs. The traffic generation parameters are defined in a generic "music audio data traffic model" proposed in [23] and summarized in Table 1. This is a stochastic model that emulates the audio data production from a music instrument or singer based on normal distribution around the tempo of $120 \mathrm{bpm}[24,25]$. OPNET Modeler 17.1 network simulation platform it is also used for this study. Figure 9 provides a graphical representation of OPNET's hierarchical architecture for a 56 audio STAs scenario, with additional data STAs in the network. The statistics collected during the simulation are Throughput and Overall End-to-End Delay.

Before proceeding with the analysis of the results it is useful to clarify throughput measurement in broadcasting. For this purpose, a wireless network with $n$ STAs where each of them produces a data load of $A i$ (bit/s) is assumed. If we consider all transmissions to be successful over a period $\Delta t$, each STA will finally receive during this period a total load $T_{\Delta t}$ which is described by (11).

$$
T_{\Delta t}=A_{1}+A_{2}+\cdots+A_{i-1}+A_{i+1}+\cdots+A_{n}=(n-1) \times A_{i}
$$

Equation (11) shows that a much higher overall throughput than the produced data should be expected as every STA in the network receives all the data produced from all other STAs except its own transmitted data. Having $n$ STAs in the network, the maximum theoretical throughput in the entire network $\Sigma\left(T_{\Delta t}\right)$ will be given by:

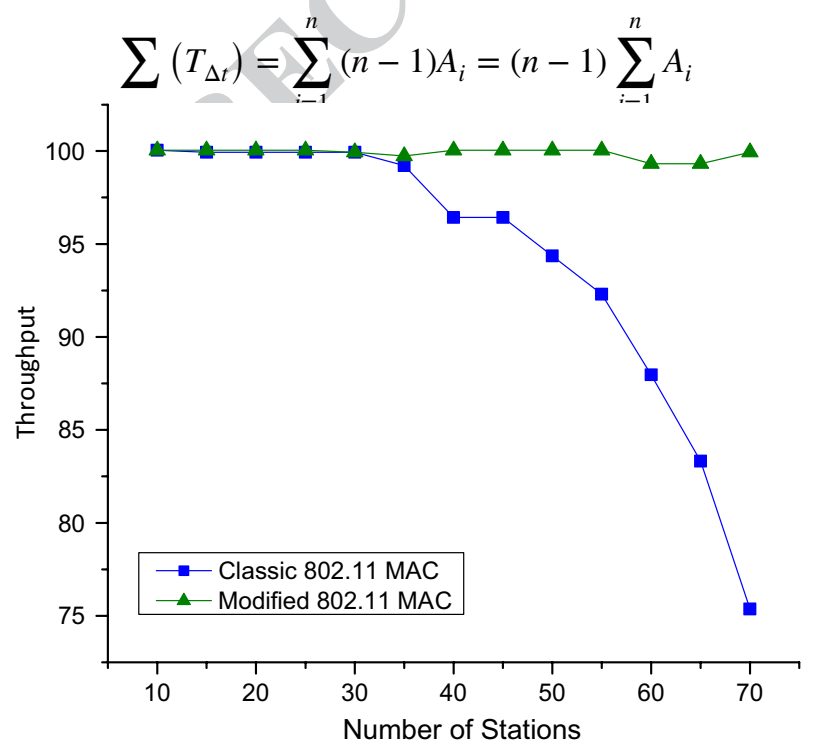

Fig. 10 Throughput performance, (Classic 802.11 vs. EBNA modified MAC) 
If the data load $A$, that is produced from all STAs is equal, then Eq. (12) can be simplified as follows:

$$
\Sigma\left(T_{\Delta t}\right)=n \times[(n-1) \times A]
$$

Equation (13) shows again that the actual throughput of the entire network, in case of broadcasting, is higher than the overall broadcasted data load at any given time.

The graph in Fig. 10 shows a comparison between the classic and modified IEEE 802.11 MAC as it is resulting from these simulations. It is clearly shown that the EBNA modified congestion control mechanism is able to handle a vast number of broadcasting STAs comparing to the classic 802.11 which causes a significant number of collisions when the number of STAs in the network increases.

Figure 11 shows the overall end-to-end delay for both the EBNA modified and the classic 802.11 MAC. It is shown here that by using the EBNA algorithm we significantly increase the overall end-to-end delay in the network. This is due to two main causes. First, it is because of the additional traffic added in the network by using the CTS-to-Self protection mechanism, discussed in the previous section. Second, it is because of the linear increase of $\mathrm{CW}$ window that results when the EBNA algorithm is used.

When EBNA is applied, each increase on the network's population causes in turn a linear increase of the $\mathrm{CW}$ as it shown from (6). A larger $\mathrm{CW}$ allows higher waiting time values to be used and therefore increases delay.

This delay can be considered appropriate for media streaming but is marginally acceptable for networks that require real time audio delivery. As it is resulting from [26] and from the general audio engineering practices, an approximately $10 \mathrm{~ms}$ average delay would be required, for a 60 STAs scenario, in order for a wireless audio network to be considered functional. Therefore, an improvement regarding delay

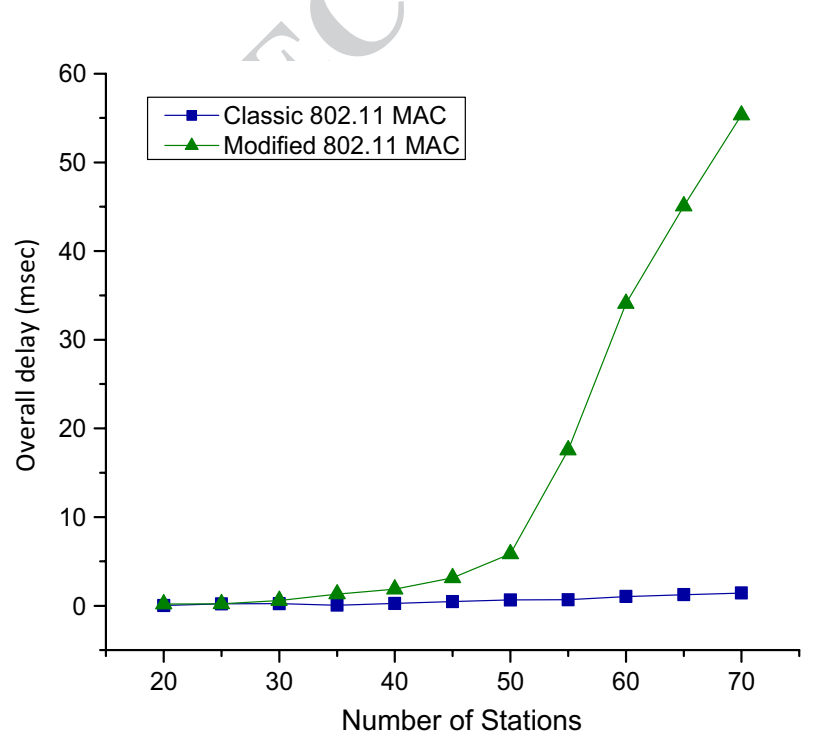

Fig. 11 Overall end-to-end delay for classic and EBNA modified 802.11 MAC 
performance is needed in order for the EBNA concept to be used in wireless audio networking.

\section{Hybrid EBNA algorithm}

The implementation of EBNA in the previous section leads to a number of significant conclusions. Initially, it is shown that collisions can be eliminated by using this method. However, this technique results in an increase of the overall delay due to the linear increase of $\mathrm{CW}$, which is proportional to the number of STAs in the network. On the other hand, the performance of classic 802.11 when it comes to throughput and overall delay is satisfactory especially when only few STAs are broadcasting. [27]. When a small number of STAs is broadcasting in a wireless network, the probability of collision is low (Fig. 2). Taking into account that the $\mathrm{CW}$ is also small, classic 802.11 achieves equivalent throughput values with the EBNA, but with a significantly lower delay.

The EBNA algorithm was designed for fully saturated wireless networks, where all STAs have data to broadcast at all times. For that reason, the algorithm reserves always an exclusive pair of backoff values for each STA. That means that for each new STA joining the network, the size of CW increases by two values. This results in a relatively large CW, especially when the number of STAs increases. However, musical data production has a stochastic nature. That means that during a session there are several time intervals where STAs have no data to broadcast. Hence, as the size of $\mathrm{CW}$ remain constant, unnecessary long waiting times are added by the congestion control algorithm. The possibility of monitoring the network and applying the EBNA concept using a dynamic CW, only for active STAs, will result in a lower overall delay.

Taking into account the above characteristics, a Hybrid-EBNA (H-EBNA) algorithm is proposed in this section. This algorithm monitors the activity of all STAs in the network, calculates the probability of collisions and regulates congestion by automatically switching between the classical IEEE 802.11 and the EBNA MAC. In addition, when EBNA is selected, identifies the number of active STAs in the network and implements a dynamic CW in order to keep average backoff time in the lower possible levels. The operating principle of the H-EBNA algorithm within each STA is described below:

- The algorithm constantly monitors the network's activity.

- When the STA has a packet to broadcast, the algorithm identifies the number of active STAs in the network.

- If the probability of collision using the classic IEEE 802.11 is low, the algorithm selects this method to access the medium and broadcast its data.

- If the probability of collision using the classic IEEE 802.11 is high, the algorithm switches to the H-EBNA MAC. Then it accesses the medium and broadcasts its data.

- When H-EBNA is selected, the enhanced CTS-to-Self-protection mechanism is also applied by default. 
The H-EBNA algorithm complies with the general concept of the distributed coordination which characterizes the IEEE 802.11 standard. Therefore, the algorithm is implemented independently in each individual STA in the network. That gives flexibility in the setup and maintenance of the network which is a fundamental requirement in audio networking.

\subsection{Generation and maintenance of the List-of-STAs}

When the H-EBNA modified MAC is implemented, the first action of the algorithm is to generate a record that contains all STAs in the network. STAs associating with the wireless network obtain automatically a STID. When a CTS-to-Self is transmitted prior to each data transmission, The STID of the broadcasting STA is included in the body of this message. With this mechanism all STAs after their first access to the medium register their STIDs with all peer STAs in the network.

Every STA that starts operating within the network generates a static list called "General List of STAs". Each record on this list contains in ascending order a STID and the timestamp of the most recently broadcasted CTS-to-Self message, coming from the STA with this particular ID. During the session, when a CTS-to-Self message with a specific STID is received, the algorithm updates the timestamp for this specific ID in the "General List of STAs". This technique also resolves the issue of synchronization between STAs in the network. Individual STAs can use their own timers as no clock distributions is required. As it will be discussed later in this paper, for the implementation of H-EBNA, time differences rather than absolute time values are required.

\subsection{Defining the active STAs in the network}

The next step for the implementation of H-EBNA is to identify the active STAs in the network. This procedure is executed independently in each STA prior to each transmission. When a STA has a packet to transmit, the algorithm uses the set of information stored in the "General List of STAs" to generate the sub-set of active STAs in the network. This sub-set has the form of a dynamic table and it is called "List of Active STAs". In order to create this second list, the algorithm subtracts from the current time $\left(T_{C}\right)$ the time $\left(T_{A}\right)$ that the last CTS-to-Self has arrived for each specific STID. Then it compares the result $\left(T_{D}\right)$ with a predefined threshold value $T_{\text {threshold }}$. The decision whether an arbitrary STA $k$ will be considered as active or inactive is taken as follows:

$$
T D_{k}=\left[T_{C}\right]-\left[T_{A}\right]_{k}
$$

$$
\text { For : } \quad \begin{aligned}
& T D_{k} \geq T_{\text {threshold }} \quad \text { STA = Active } \\
& T D_{k}<T_{\text {threshold }} \text { STA = Inactive }
\end{aligned}
$$


If the IEEE $802.11 \mathrm{~g}$ with a transmission rate of $54 \mathrm{Mbps}$ is used, each bit requires a period of $1.8 \times 10^{-8} \mathrm{~s}$ in order to be transmitted. Therefore, a data byte will require a transmission time of $1.481 \times 10^{-7} \mathrm{~s}$. The H-EBNA is implemented using constant maximum packet size and therefore each packet with a size of 2234 byte will need a Packer-Transfer-Time (PTT) PTT $=3.31 \times 10^{-4} \mathrm{~s}$. The regular size of a CTS-to-Self message is 14 bytes and it is also transmitted with the $54 \mathrm{Mbps}$ bit rate. Therefore, the CTS-to-Self Transmission Time (CTSTT) will be CTSTT $=2.0735 \times 10^{-6} \mathrm{~s}$. We denote as "round time" the minimum time period required for all STAs in a network to transmit a packet. The maximum expected population in a wireless audio network is 60 STAs. Therefore, the round time in a 60 STAs scenario $\left(\right.$ Round $\left._{60}\right)$, will be:

$$
\text { Round }_{60}=60 \times(P T T+C T S T T+\text { DIFS }+ \text { SIFS })
$$

From (15) and for SIFS $=10 \times 10^{-6} \mathrm{~s}$ and $D I F S=50 \times 10^{-6} \mathrm{~s}$, the one round timeframe is calculated to be $0.01,998 \mathrm{~s}$. In the implementation of H-EBNA we adjust the value of $T_{\text {threshold }}$ in order for each STA to be given a "three rounds opportunity" to transmit a packet using the maximum round time value $\left(\right.$ Round $\left._{60}\right)$. This gives consequently a $T_{\text {threshold }-60}=0.05995 \mathrm{~s}$. Using the above described scheme, we actually give to all STAs in the network a probability of at least $300 \%$ to gain access to the medium and complete a packet broadcast. STAs that fail to achieve this target are considered inactive and their ID is removed from the "List of Active STAs". Figure 12 shows an example of the "General List of STAs" and its product "List of Active STAs" as described above.

\subsection{Switching between classic and EBNA 802.11 MAC}

After the generation of the "List of Active STAs", which is updated every time a packet has to be transmitted, the algorithm has to decide whether to use the classic IEEE 802.11 or the H-EBNA modified MAC for accessing the medium. It is important to note here that, although there is not a central control, we are expecting all STAs to have a coordinated transition between H-EBNA and classic 802.11. This is

\section{General List of STAs}

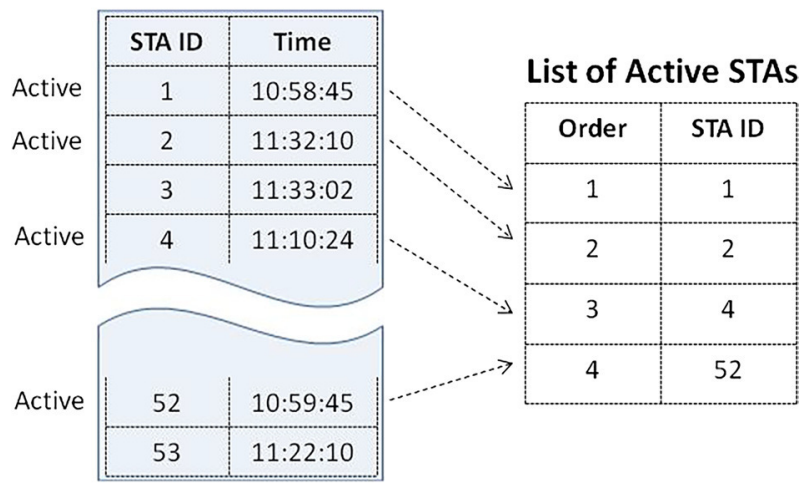

Fig. 12 The general list of all STAs and the list of active STAs (Example) 
481

because all STAs are monitoring the network simultaneously and they collect identical information when it comes to the data traffic.

The transition between H-EBNA and classic 802.11 is based on a critical number of active STAs $\left(N_{T}\right)$ which consequently results a critical probability of collision $\left(p_{T}\right)$. This probability of collision is defined indirectly by the user who is required to declare a maximum acceptable packet loss rate. If $P$ is the percent of the maximum acceptable packet loss rate, the maximum acceptable probability of collision will be $p_{T}=\mathrm{P} / 100$. Using (5) we can obtain an expression for the critical number of active STAs as follows:

$$
p_{T}=1-\left(1-\frac{1}{C W}\right)^{N_{T}-1}
$$

$$
\therefore N_{T}=\frac{\log \left(1-p_{T}\right)}{\log \left(1-\frac{1}{C W}\right)}-1
$$

The algorithm allows the user to set manually the parameters $P$ and $\mathrm{CW}$ when the network is formed, however these values will remain constant during the operation of the network. The process of switching between MACs is regulated by the following rule:

$$
\begin{array}{r}
N>N_{T} \text { EBNA MAC } \\
N \leq N_{T} \text { Classic 802.11 MAC }
\end{array}
$$

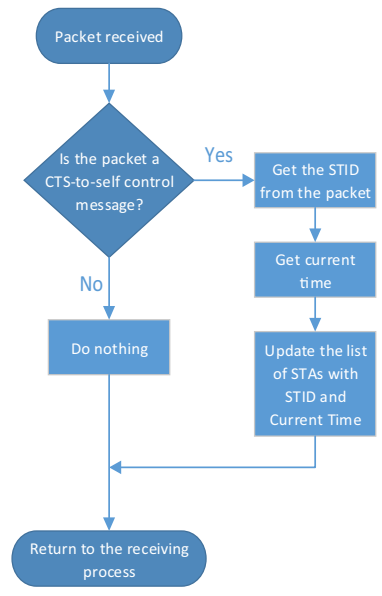

(a)

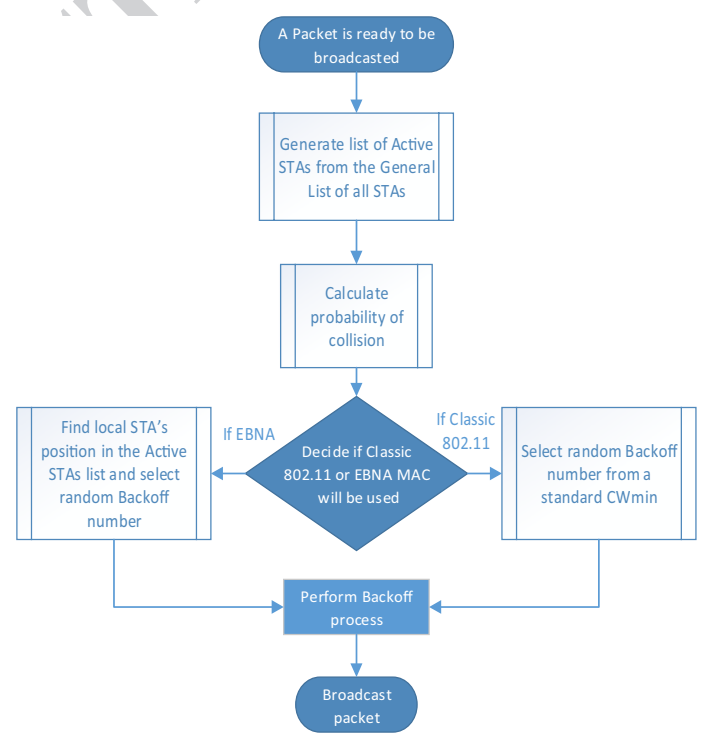

(b)

Fig. 13 Operation of H-EBNA algorithm at the receiver (a) and the transmitter (b) 
According to (18), whenever the population of active STAs in the network becomes greater than the critical number $N_{T}$, the algorithm switches to the H-EBNA mechanism. When the population of active STAs drops, it switches back to the classic IEEE 802.11. Using this technique, the algorithm manages to maintain a high throughput while keeping the overall delay in lower levels.

The flowchart in Fig. 13a shows the network monitoring process at the receiver. The flowchart in Fig. 13.b shows the action taken by the algorithm at the transmitter. Here, when a packet is to be transmitted, the algorithm calculates the probability of collision taking also into account the users setting and decides whether to use EBNA or classic 802.11.

\subsection{H-EBNA (simulation and results)}

The simulation characteristics, the topology, the traffic generation parameters and the collected results are the same as those in the EBNA simulation shown in Sect. 4.1.

Figure 14 shows the overall throughput, measured during the simulation for a 60 STAs network. This graph contains the results from the three congestion methods discussed in this paper. This 60 STAs scenario is a complete stress test of the H-EBNA algorithm when it comes to throughput performance because wireless audio network with this population is considered as the desirable commercial target.

This graph shows that both H-EBNA and basic EBNA are performing equally well when it comes to throughput. They also both give better throughput results than classic IEEE 802.11 which fail to eliminate collisions in multi-broadcasting environment.

However, the most significant difference is illustrated in Fig. 15 where the average delay is presented. It is shown here that the implementation of H-EBNA achieves

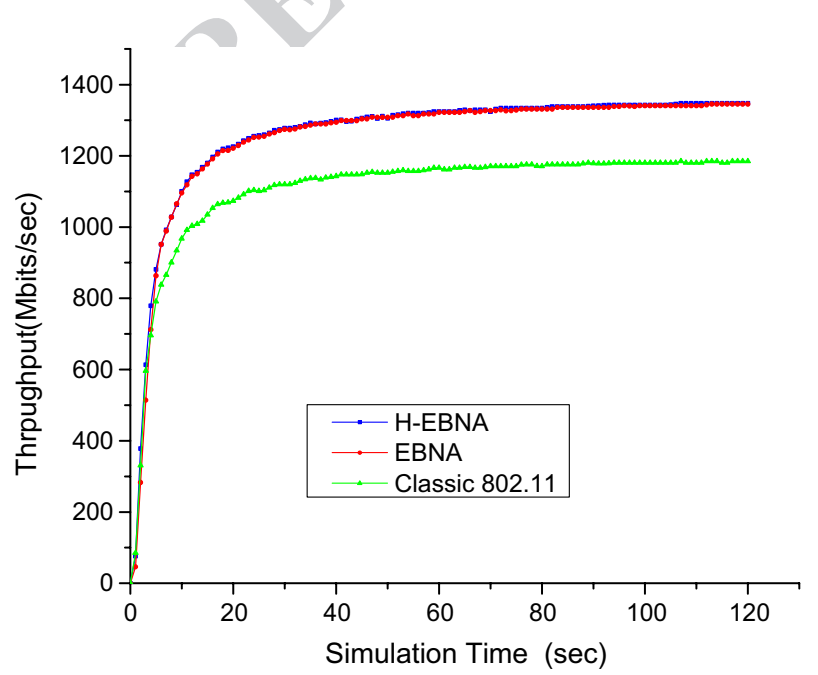

Fig. 14 Throughput performance, WLAN of 60 STAs 


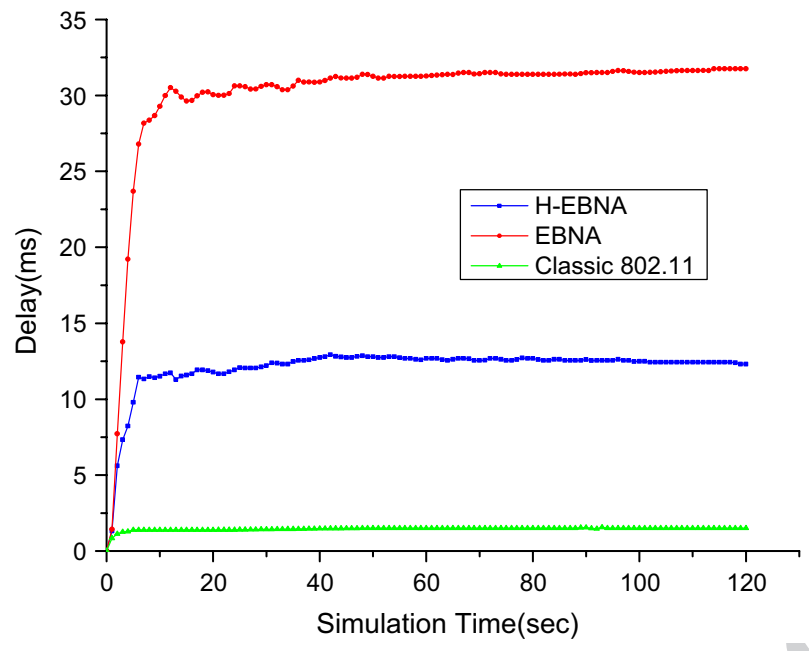

Fig. 15 Average end-to-end delay, WLAN of 60 STAs

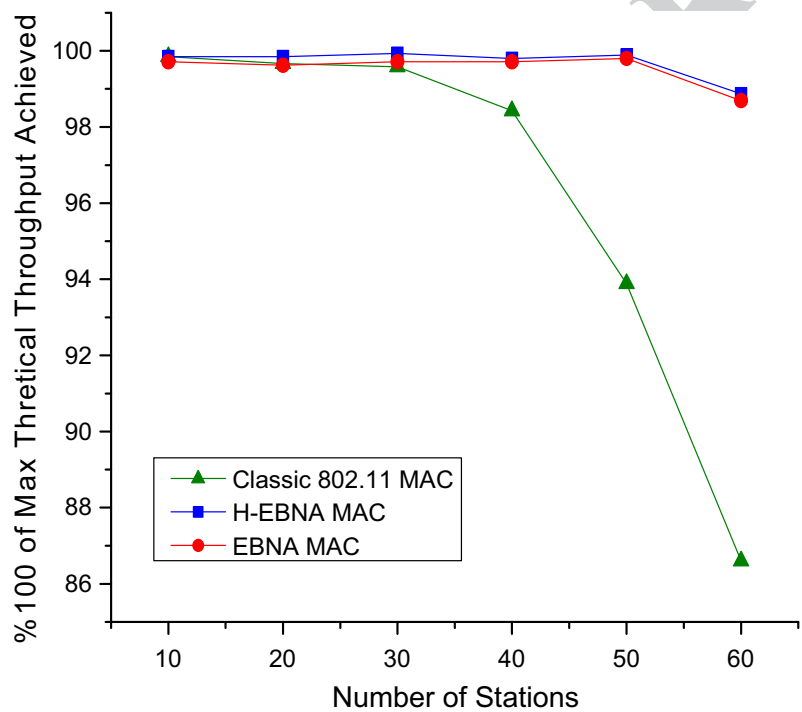

Fig. 16 Throughput performance, (Classic 802.11, H-EBNA and EBNA modified MAC) 


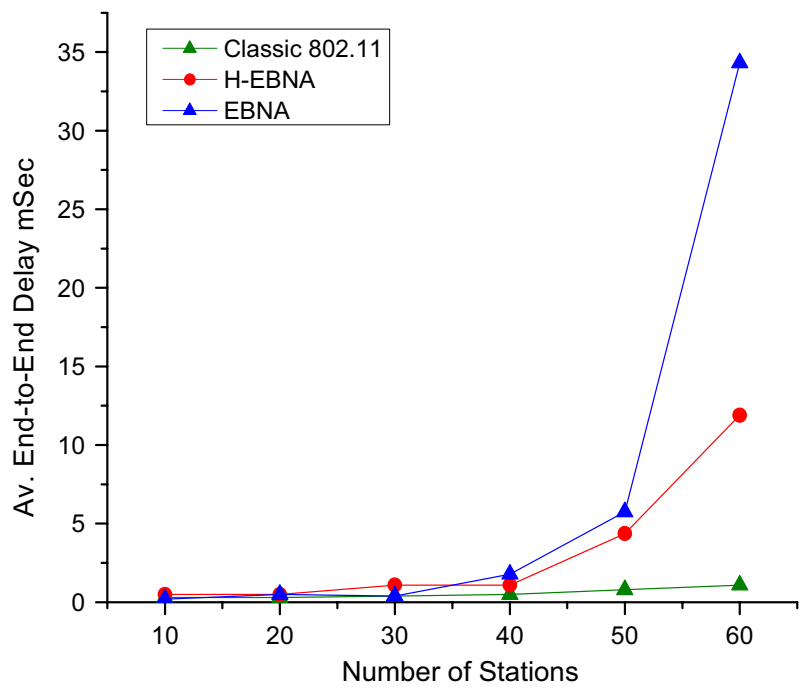

Fig. 17 End-to-end delay, (Classic 802.11, H-EBNA and EBNA modified MAC)

significantly lower average delay (approximately $12 \mathrm{~ms}$ ), which is also within the commonly accepted limits for real time audio networking applications.

Figures 16 and 17 show the Throughput and Delay performance respectively, collected from all the simulation scenarios. Here it is also shown that basic EBNA and H-EBNA are equally successful when it comes to throughput, however, H-EBNA significantly outweighs when it comes to delay. It is important to mention here that the packet loss observed in scenarios of 55 STAs and above it is caused due to the buffer overflow within the STAs and not because of the collisions.

\section{Conclusions}

In this paper we propose a complete set of modifications for the IEEE 802.11 standard in order to be able to support the implementation of wireless audio networks.

Audio networks represent a dynamically evolving sector within the audio engineering industry and their expansion in the wireless notworking domain has significant potential. The amendment proposed in this work aims to overcome the fundamental problems that prevent the use of the existing wireless technologies toward this direction. We focus our effort on the IEEE 802.11 standard, which is widespread, efficient, inexpensive and well adopted by the market.

The proposed set of amendments consists of two main categories of modifications. The first one provides a collision protection mechanism for broadcasting by extending the use of the CTS-to-Self message. The second one resolves the congestion control issue by introducing an EBNA method that minimizes the probability of collision in broadcasting for a finite, known number of STAs in a wireless 
ad-hoc network. Moreover, an extended version of this method called hybrid-EBNA is proposed.

Using this method, STAs in the network are able to monitor the data traffic and switch between classic 802.11 and EBNA, achieving this way high throughput and low delay.

The above amendments were tested in a simulation environment. The results showed that this modified IEEE 802.11 MAC mechanism achieves the necessary performance when it comes to throughput and delay, and therefore it can be used as the networking platform for the commercial development of wireless audio networks.

\section{References}

1. Gross, K.: Audio networking: applications and requirements. J. Audio Eng. Soc. 54(1/2), 62-66 (2006)

2. AES67.: Standard for audio applications of networks-high-performance streaming audio-over-IP interoperability. Audio Engineering Society (2013)

3. Ulema, M., Nogueira, J.M., Kozbe, B.: Management of wireless ad hoc networks and wireless sensor networks. J. Netw. Syst. Manag. 14(3), 327-333 (2006)

4. Setton, E., Yoo, T., Zhu, X., Goldsmith, A., Girod, B.: Cross-layer design of ad hoc networks for real-time video streaming. IEEE Wirel. Commun. 12(4), 59-65 (2005)

5. Liu, H., Yang, H., Wang, Y., Wang, B., Yuantao, G.: CAR: coding-aware opportunistic routing for unicast traffic in wireless mesh networks. J. Netw. Syst. Manag. 23(4), 1104-1124 (2015)

6. Dai, B., Wei, Yu.: Sparse beamforming and user-centric clustering for downlink cloud radio access network. IEEE Access. 2, 1326-1339 (2014)

7. Rong, B., Sun, S., Kadoch, M.: Traffic prediction for reliable and resilient video communications over multi-location WMNs. J. Netw. Syst. Manag. 24(3), 516-533 (2016)

8. Chen, W.-K.: AES-technical committee on network audio systems, white paper, best practices in network audio. In: Audio Engineering Society, Linear Networks and Systems, pp. 123-135. Wadsworth, Belmont, CA

9. Karthikeyan, N., Palanisamy, V., Duraiswamy, K.: Performance comparison of broadcasting methods in mobile ad hoc network. Int. J. Fut. Gen. Commun. Netw. 2(2), 47-58 (2009)

10. Oh, B.J., Chang, W.C.: A cross-layer approach to multichannel MAC protocol design for video streaming over wireless ad hoc networks. IEEE Trans. Multimed. 11(6), 1052-1061 (2009)

11. Lipman, J., Liu, H., Stojmenovic, I.: Broadcast in ad hoc networks. In: Guide to Wireless Ad Hoc Networks, pp. 121-150. Springer, London (2009)

12. Williams, B., Camp, T.: Comparison of broadcasting techniques for mobile ad hoc networks. In: Proceedings of the 3rd ACM International Symposium on Mobile Ad Hoc Networking and Computing, pp. 194-205. ACM (2002)

13. Floros, A., Karoubalis, T.: Delivering high-quality audio over WLANs. In: Audio Eng. Soc. 116th Convention, Berlin, vol. 5996 (2004)

14. Fluke networks White Paper: Ensuring 802.11n and $802.11 \mathrm{a} / \mathrm{b} / \mathrm{g}$ Compatibility (2011)

15. Guo, Y., Liu, Y., Liu, G., Wang, X.: Pair-wise collision-based underwater time synchronization. J. Netw. Syst. Manage. 24(4), 813-833 (2016)

16. Vu, H.L., Sakurai, T.: Collision probability in saturated IEEE 802.11 networks. In: Australian Telecommunication Networks and Applications Conference, Australia (2006)

17. Greco, C., Cagnazzo, M., Pesquet-Popescu, B.: Low-latency video streaming with congestion control in mobile ad-hoc networks. IEEE Trans. Multimedia 14(4), 1337-1350 (2012)

18. Lee, J.-W., Chiang, M., Calderbank, A.R.: Utility-optimal random-access control. IEEE Trans.AQ2 Wireless Commun. 6(7), 2741-2751 (2007)

19. Chatfield, C.: Statistics for technology: a course in applied statistics. Routledge, Abingdon (2018) 
20. Zhang, T., Lei, L., Zhou, J., Qi, L.: Collision probability analysis in multi-hop ad hoc networks. In: 8th International Conference on Wireless Communications, Networking and Mobile Computing (WiCOM), 2012, pp. 1-4. IEEE (2012)

21. Williams, B., Camp, T.: Comparison of broadcasting techniques for mobile ad hoc networks. In: Proceedings of the 3rd ACM international symposium on mobile ad hoc networking and computing, pp. 194-205. ACM (2002)

22. Chousidis, C., Nilavalan, R., Laurentiu, L.: Expanding the use of CTS-to-self mechanism for reliable broadcasting on IEEE 802.11 networks. In: International Wireless Communications and Mobile Computing Conference (IWCMC), 2014, pp. 1051-1056. IEEE (2014)

23. Chousidis, C., Nilavalan, R., Floros, A.: Enhancement of IEEE 802.11 in handling multiple broadcasting audio data in wireless ad-hoc networks. J. Audio Eng. Soc. 61(4), 165-173 (2013)

24. Moelants, D., McKinney, M.: Tempo perception and musical content: What makes a piece fast, slow or temporally ambiguous. In: Proceedings of the 8th International Conference on Music Perception and Cognition, pp. 558-562 (2004)

25. Jindal, P., Singh, B.: Security-performance tradeoffs in a class of wireless network scenarios. J. Netw. Syst. Manage. 25(1), 83-121 (2017)

26. AES67: Standard for audio applications of networks-High-performance streaming audio-over-IP interoperability. Audio Engineering Society (2013)

27. Chousidis, C., Nilavalan, R.: Modifying the IEEE 802.11 MAC to improve performance of multiple broadcasting of multimedia data in wireless ad-hoc networks. Int. J. Adv. Comput. Sci. Appl. 3(4), 70-77 (2012)

Publisher's Note Springer Nature remains neutral with regard to jurisdictional claims in published maps and institutional affiliations.

Christos Chousidis received the M.Phil and the Ph.D. from the Department of Electronic and Computer Engineering, Brunel University London, in 2006 and 2014, respectively. He is currently a Senior Lecturer in Applied Sound Engineering with the School of Computing and Engineering at the University of West London, UK. His current research interests include wireless audio networks and bioelectric sensors for human voice capturing. He is a member of IEEE and a member of the technical committee on network audio systems (TC-NAS) of the Audio Engineering Society.

Ioana Pisica received the M.Sc. degree in information systems from the Academy of Economic Studies, Bucharest, and the Ph.D. degree in intelligent energy networks from the University Politehnica of Bucharest. She is currently a Senior Lecturer of Power Systems with the Department of Electronic and Computer Engineering, Brunel University London. Her research interests include data analytics for energy systems, machine learning for power systems control, power quality, smart metering, and ICT infrastructures for future power networks and energy efficiency. She is a member of IET-UK and IEEE.

Zhengwen Huang received a B.Sc. from the University of Science and Technology, Hefei, China, an M.Sc. from King's College London, London, UK, and a Ph.D. degree from the Department of Electronic and Computer Engineering, Brunel University London, UK, in 2014. Hi is currently a Lecturer in Electronic and Computer Engineering in the Brunel University London. His current research interests include evolutionary algorithms (gene expression programming and genetic programming) and data engineering. 